\title{
On The Failure of The Linkage Principle in Multi-Unit Auctions*
}

\author{
Motty Perry \\ Center for Rationality, The Hebrew University \\ of Jerusalem, Jerusalem, Israel \\ email: motty@sunset.huji.ac.il \\ Philip J. Reny \\ Department of Economics, University of Pittsburgh \\ Pittsburgh, PA 15260 \\ email: reny+@pitt.edu
}

August 1997

\begin{abstract}
It is shown that the linkage principle (Milgrom and Weber (1982)) does not extend to the multi-unit auction setting. An analysis of the equilibium bidding strategies is carried out for the general two-agent/two-unit Vickrey auction in order to provide economic insight into the nature of the failure. In addition, an explicit counterexample is provided.
\end{abstract}

* Both authors acknowledge support from the Binational Science Foudation (grant\#9500023/1). Reny also acknowledges support from the National Science Foundation (grant\# SBR-970932), and the University of Pittsburgh's Faculty of Arts and Sciences. 


\section{Introduction}

Milgrom and Weber (1982) provide a number of important results on the revenueranking of various single-unit auction forms under incomplete information. Their analysis uncovered the so-called linkage principle which states that, on average, revenues are enhanced by always providing the bidders with as much information as possible about the value of the good. The linkage principle has come to be considered one of the fundamental lessons provided by auction theory.

The significance and general acceptance of the linkage principle as a guide to auction design, even in contexts beyond single-unit auctions, is highlighted by the recent design of the spectral auction held by the FCC, which contains an open-auction component. Although the experts agreed that collusion among the bidders (which ultimately did occur; The Economist May 17, 1997, p.86) is more easily sustained within an open auction, in the end the faith placed in the linkage principle outweighed this concern and an open auction format was employed. Indeed, according to McMillan (1994), the experts "judged [the negative collusion effect] to be outweighed by the bidders' ability to learn from others' bids in the open auction."

Despite the faith placed in the linkage principle, we shall show that it does not generally hold in settings beyond single-unit auctions. Indeed, it is enough to consider a two-unit Vickrey (1961) auction to demonstrate this. By characterizing the equilibrium strategies in this simplest of multi-unit settings, we both illustrate the economics behind the failure of the linkage principle and provide an explicit counterexample. ${ }^{1}$ Thus, the linkage principle can be added to the list of results from mechanism design and auction theory which fail to extend from the singleunit/single-dimensional context to the multi-unit/multi-dimensional one (see, for example, Armstrong (1996)).

The rest of the paper is organized as follows. Section 2 describes the model and provides the general structure of equilibrium bidding strategies in a twoagent/two-unit Vickrey auction. This theoretical analysis provides the basis for understanding the economics behind the failure of the linkage principle. Section

\footnotetext{
${ }^{1}$ In a recent paper, Ausubel (1997) establishes the linkage principle in a multi-unit Vickrey auction setting. However, in his model, agents are retsricted to having constant marginal valuations (i.e. flat demand). This tends to move the analysis toward that employed in the single-unit case.
} 
3 introduces into the model private information held by the seller. Section 4 draws on the analysis of Sections 2 and 3 to explain why the linkage principle fails. Section 5 contains an explicit counterexample, and Section 6 provides some concluding remarks.

\section{The Model}

A seller wishes to sell two units of a single good. The two units are identical and each is indivisible. There are two agents. Each agent receives a real-valued signal, which might, for example, be a measure of the good's quality. The agents are symmetric in that if $x$ is one agent's signal and $y$ is the other's, then $m_{k}(x, y)$ denotes the one's marginal valuation for the $k^{\text {th }}$ unit of the good, $k=1,2$. The signals are distributed on the unit square with density $f(x, y)$, which is symmetric in its arguments. Each agent is informed of his own signal, but not that of the other agent. Consequently, not only does each agent have incomplete information regarding the other agent's marginal valuations, he also has incomplete information regarding his own marginal valuations.

We shall assume that each $m_{k}(\cdot, \cdot)$ is continuous, non-decreasing in each argument, and that $m_{1}(x, y) \geq m_{2}(x, y)$ for all $x, y$.

\subsection{The Two-Unit/Two-Agent Vickrey Auction}

Consider now the Vickrey (1961) auction for the case of two agents and two units. ${ }^{2}$ Each agent submits a pair of bids. Among the four submitted bids, the highest two are deemed "winning," and the lowest two "losing." If a single agent submits both winning bids, he is awarded both units and he pays the seller the sum of the other agent's bids. If each agent submits a winning bid, then each is awarded one unit of the good and each agent pays the seller the other agent's losing bid.

Under the above rules, one can identify the larger of the two bids submitted by an agent as his bid for a first unit, and the lower of the two bids as that for a second unit. Thus, we can think of the agents as submitting a pair of bids $\left(b_{1}, b_{2}\right)$, with $b_{1} \geq b_{2}$ where $b_{k}$ is interpreted as the agent's bid for a $k^{\text {th }}$ unit.

In order to understand the economics behind the failure of the linkage principle, we now derive equilibrium equations for this auction. We freely assume

\footnotetext{
${ }^{2}$ But note that in Vickrey's model, each agent has complete information regarding his own marginal valuations.
} 
differentiability and other properties along the way in order to focus attention on the main ideas.

So, let us focus attention on symmetric pure strategy equilibria which are differentiable and strictly increasing in the agent's signals, and in which an agent's bid for a first unit is never less than his bid for a second.

To characterize these, we begin by supposing that $\left(\hat{b}_{1}(\cdot), \hat{b}_{2}(\cdot)\right)$ constitutes such an equilibrium strategy for each bidder, where $\hat{b}_{k}(x)$ denotes an agent's bid for a $k^{\text {th }}$ unit when his signal is $x$. Consider then agent 1 's expected utility from submitting a pair of bids $b_{1}>b_{2}>0$. Given that the equilibrium strategy is employed by agent 2 , agent 1 's expected utility is:

$$
\begin{gathered}
U\left(b_{1}, b_{2} \mid x\right)=E\left\{\left(m_{1}(X, Y)-\hat{b}_{2}(Y)\right) \mathbf{1}_{\left\{b_{1}>\hat{b}_{2}(Y)\right\}} \mid X=x\right\}+ \\
E\left\{\left(m_{2}(X, Y)-\hat{b}_{1}(Y)\right) \mathbf{1}_{\left\{b_{2}>\hat{b}_{1}(Y)\right\}} \mid X=x\right\} \\
=\int_{0}^{\hat{b}_{2}^{-1}\left(b_{1}\right)}\left(m_{1}(x, \alpha)-\hat{b}_{2}(\alpha)\right) f(\alpha \mid x) d \alpha+\int_{0}^{\hat{b}_{1}^{-1}\left(b_{2}\right)}\left(m_{2}(x, \alpha)-\hat{b}_{1}(\alpha)\right) f(\alpha \mid x) d \alpha
\end{gathered}
$$

Differentiating this expression with respect to the $b_{i}$ 's yields the following firstorder conditions. ${ }^{3}$

$$
\begin{aligned}
& \frac{\partial U}{\partial b_{1}}=\left[m_{1}\left(x, \hat{b}_{2}^{-1}\left(b_{1}\right)-b_{1}\right] \frac{f\left(\hat{b}_{2}^{-1}\left(b_{1}\right) \mid x\right)}{\hat{b}_{2}^{\prime}\left(\hat{b}_{2}^{-1}\left(b_{1}\right)\right)}=0\right. \\
& \frac{\partial U}{\partial b_{2}}=\left[m_{2}\left(x, \hat{b}_{1}^{-1}\left(b_{2}\right)-b_{2}\right] \frac{f\left(\hat{b}_{1}^{-1}\left(b_{2}\right) \mid x\right)}{\hat{b}_{1}^{\prime}\left(\hat{b}_{1}^{-1}\left(b_{2}\right)\right)}=0\right.
\end{aligned}
$$

Since in equilibrium, the first-order conditions must hold for every equilibrium bid, we obtain the following equilibrium conditions. For every $x \in[0,1]$ :

$$
\begin{aligned}
& \hat{b}_{1}(x)=m_{1}\left(x, \hat{b}_{2}^{-1}\left(\hat{b}_{1}(x)\right)\right), \text { and } \\
& \hat{b}_{2}(x)=m_{2}\left(x, \hat{b}_{1}^{-1}\left(\hat{b}_{2}(x)\right)\right)
\end{aligned}
$$

\footnotetext{
${ }^{3}$ This assumes an interior solution and that the bids are in the appropriate ranges.
} 
The theorem below establishes that conditions (??) and (??) are sufficient for $\left(\hat{b}_{1}(\cdot), \hat{b}_{2}(\cdot)\right)$ to constitute an equilibrium. ${ }^{4}$ The proof can be found in the Appendix.

Theorem 2.1. Suppose that $\hat{b}_{1}(\cdot) \geq \hat{b}_{2}(\cdot)$ are strictly increasing on $[0,1]$, and that they satisfy (??) and (??). Then each agent employing the strategy $\left(\hat{b}_{1}(\cdot), \hat{b}_{2}(\cdot)\right)$ constitutes a symmetric equilibrium of the two-unit/two-agent Vickrey auction.

Equations (??) and (??) are central to understanding the failure of the linkage principle. Consequently, we now take a moment to discuss them.

Suppose that agent 1's signal is $x$, and 2's is $y$. Recall that according to the rules of the auction, 1's first-unit bid (i.e. $\hat{b}_{1}(x)$ ) is winning when it is above 2 's second-unit bid (i.e. $\left.\hat{b}_{2}(y)\right)$. According to (??), 1's first-unit bid is equal to his first-unit value when 2's signal is as high as possible, yet consistent with a winning first-unit bid of agent 1. Consequently, when 1's first-unit bid is winning (resp., losing), the signal he "attributes" to agent 2 (for the purpose of obtaining a first-unit bid) is above (resp., below) 2's true signal. ${ }^{5}$

Similarly, 1's second-unit bid is winning when it is above 2's first-unit bid. According to (??), 1's second-unit bid is equal to his second-unit value when 2's signal is as high as possible, yet consistent with a winning second-unit bid of agent 1 . Therefore, when 1's second-unit bid is winning (resp., losing), the signal he "attributes" to agent 2 (for the purpose of obtaining a second-unit bid) is above (resp., below) 2's true signal.

The essential point of all of this is summarized by the following "biased signal" property

\footnotetext{
${ }^{4}$ Suitable restrictions on the $m_{k}(\cdot, \cdot)$ functions guarantee that equations (??) and (??) possess a solution. For example, the following additional conditions (which many examples satisfy) suffice: $m_{1}(0,0)=m_{2}(0,0), m_{1}(1,1)=m_{2}(1,1)$, and $m_{1}(x, y)-m_{2}(y, x)$ is strictly increasing in $x$ and strictly decreasing in $y$. The condition on the difference expresses the idea that one's own signal affects one's value more than others' signals do. Thus, the existence of an equilibrium of the kind we consider here can be established quite generally.

${ }^{5}$ For example, suppose that 1's signal is $x$ and that his first-unit bid is winning. Then it must be the case that $\hat{b}_{1}(x)$ exceeds agent 2's second-unit bid, $\hat{b}_{2}(y)$, where $y$ is agent 2's signal. Consequently, 1 's estimate, $\hat{y}=\hat{b}_{2}^{-1}\left(\hat{b}_{1}(x)\right)$, of 2's signal for the purpose of obtaining a first-unit bid is above 2's true signal since $\hat{y}=\hat{b}_{2}^{-1}\left(\hat{b}_{1}(x)\right)>y$.
} 
In a Vickrey auction, winning bids (both first and second-unit) arise when the opponent's signal is "overestimated" and losing bids arise when the opponent's signal is "underestimated." 6,7

\section{Private Information Held by the Seller}

The linkage principle asserts that when the seller possesses private information that is affiliated with the (affiliated) signals of the agents, expected revenues are enhanced (on average over the seller's information) when the seller commits to a policy of always revealing his private information. Milgrom and Weber (1982) were the first to uncover the linkage principle and to show that it holds when a single indivisible unit is sold through either a Vickrey auction or a first-price (sealed-bid) auction. In order to investigate the linkage principle in our two-unit setting, we now introduce into the model private information held by the seller.

Let $x$ and $y$ continue to denote the signals of the agents, let $z$ denote the signal obtained by the seller, and let $f(x, y, z)$ denote the joint density of the signals. It is assumed that $f$ is symmetric in $x$ and $y$ for every value of $z$. When necessary, we will write $X, Y, Z$ to denote the random variables generating the signals, and $x, y, z$ to denote their realizations. If an agent's signal is $x$, the other agent's signal is $y$, and the seller's signal is $z$, then the agent's marginal valuation for the $k^{\text {th }}$ unit is $m_{k}(x, y, z), k=1,2$, where each $m_{k}$ is continuous and non-decreasing in all arguments. As before, an agent knows only his own signal, unless the seller has committed to a policy of revealing his information, in which case each agent also knows the seller's signal, $z$. We shall consider in turn the equilibrium of the auction under the two policies in which the seller either commits to always

\footnotetext{
${ }^{6}$ This phenomenon also arises in the single-unit Vickrey auction. See Milgrom and Weber (1982). There as here, the over- and underestimation discussed here should not be identified with unsophisticated behavior. The strategies do indeed constitute an equilibrium and the agents' bidding behavior is therefore optimal. The agents can afford to employ biased estimates of the opponent's signal when contemplating their own bids because the price they pay for a unit is determined not by their own bids, but by those of the opponent.

${ }^{7}$ Since a single bidder might have both a winning first-unit bid and a losing second-unit bid, the reader might wonder how the bidder's estimate of the opponent's signal can be both an overestimate and an underestimate. The answer is that the estimate of the opponent's signal for the purpose of obtaining a first-unit bid typically differs from the estimate employed for the purpose of obtaining a second-unit bid. Indeed, the former estimate is always weakly larger than the latter.
} 
revealing his private information, or commits to never revealing it.

Consider first the case in which the seller commits to always revealing his private information. In this case, the agents' bids depend not only on their own signal, but that revealed by the seller as well, and it is easy to see that the analysis of the previous section carries through with $m_{k}(x, y)$ replaced by $m_{k}(x, y, z)$. Consequently, the equilibrium equations when the seller reveals his information are

$$
\begin{aligned}
& \hat{b}_{1}(x ; z)=m_{1}\left(x, \hat{b}_{2}^{-1}\left(\hat{b}_{1}(x ; z)\right), z\right), \text { and } \\
& \hat{b}_{2}(x ; z)=m_{2}\left(x, \hat{b}_{1}^{-1}\left(\hat{b}_{2}(x ; z)\right), z\right),
\end{aligned}
$$

where $\hat{b}_{1}^{-1}\left(\hat{b}_{2}(x ; z)\right)$ denotes the signal, $\bar{x}$, such that $\hat{b}_{1}(\bar{x} ; z)=\hat{b}_{2}(x ; z)$, and similarly for $\hat{b}_{2}^{-1}\left(\hat{b}_{1}(x ; z)\right)$.

Next, consider the case in which the seller commits to never revealing his private information. Since the seller's signal remains unknown, the agents must consider their expected marginal valuations when formulating bids. Let $\bar{m}_{k}(x, y)=$ $E\left(m_{k}(X, Y, Z) \mid X=x, Y=y\right), k=1,2$. Again, it is not hard to see that one obtains the equilibrium equations here by repeating the analysis of the previous section with $\bar{m}_{k}(x, y)$ replacing $m_{k}(x, y)$. Consequently, the equilibrium equations when the seller does not reveal his information are

$$
\begin{aligned}
& \hat{b}_{1}(x)=\bar{m}_{1}\left(x, \hat{b}_{2}^{-1}\left(\hat{b}_{1}(x)\right)\right), \text { and } \\
& \hat{b}_{2}(x)=\bar{m}_{2}\left(x, \hat{b}_{1}^{-1}\left(\hat{b}_{2}(x)\right)\right) .
\end{aligned}
$$

\section{Why the Linkage Principle Fails}

Recall the biased signal property from Section 2 .

In a Vickrey auction, winning bids (both first and second-unit) arise when the opponent's signal is "overestimated" and losing bids arise when the opponent's signal is "underestimated."

Since the analysis of Section 2 has been shown to apply equally well whether or not the seller reveals his signal, the biased signal property applies in both of 
those circumstances as well. Consider then the effect on losing bids were the seller to switch from a policy of never revealing to a policy of always revealing his signal.

When the seller does not reveal his signal, a losing bid, $\hat{b}_{2}(x)$ of agent 1 , say, is based upon an underestimate of agent 2's signal. That is, from (??), $\hat{b}_{2}(x)=E\left(m_{2}(X, Y, Z) \mid X=x, Y=\hat{y}\right)$, where $\hat{y}=\hat{b}_{1}^{-1}\left(\hat{b}_{2}(x)\right)$ is less than agent 2 's signal. ${ }^{8}$ Now, since $\hat{y}$ is below 2's true signal, and the seller's signal is affiliated with 2's, conditioning on $Y=\hat{y}$ leads to a downward bias in the estimate of the distribution of $Z$. Consequently, on average, employing the true value, $z$, of $Z$ in $m_{2}(X, Y, Z)$ will increase its expectation conditional on $Y=\hat{y}$. This indicates that revealing his signal tends (on average) to raise bids that were losing when the seller's information was not revealed.

Since the seller's revenues are precisely the sum of the two losing bids, one might think that the above provides strong intuition for the soundness of the linkage principle. However, there is another half of the story. A similar argument as above indicates that revealing his signal tends (on average) to decrease bids that were winning when the seller's information was not revealed. It is this possibility that upsets the linkage principle. Indeed, if a previously winning bid were to fall sufficiently so that it becomes a losing bid, the sum of the two new losing bids (i.e. the seller's revenue) may well be lower than the sum of the original losing bids. When this occurs, the seller's revenue falls. If this occurs sufficiently frequently, then the seller's revenues will fall on average. In the next section, we present an example illustrating these effects. Indeed, in the example, under a policy of always revealing his information the seller never raises more revenue (and with positive probability raises strictly less) than under a policy of never revealing his information.

\section{A Counterexample to the Linkage Principle}

Let $\theta, \epsilon_{x}, \epsilon_{y}, \epsilon_{z}$ be independent random variables each uniformly distributed on $[0,1]$. Define the random variables $X, Y, Z$ by $X=\theta+\epsilon_{x}, Y=\theta+\epsilon_{y}, Z=\theta+\epsilon_{z}$. Consequently, $X, Y$, and $Z$ are affiliated. As in previous sections, agent 1's signal is generated by $X$, agent 2's by $Y$, and the seller's by $Z$. Realizations of $X, Y$, and $Z$ are denoted by $x, y$, and $z$, respectively.

Let $m_{1}(x, y, z)=x+\frac{2}{3}(y+z)$, and $m_{2}(x, y, z)=x$ for all $x, y, z$. We now

\footnotetext{
${ }^{8}$ To see that $\hat{y}$ is less than agent 2 's signal, $y$, note that $\hat{b}_{2}(x)$ is a losing bid for agent 1 precisely when it is below agent 2 's first-unit bid, $\hat{b}_{1}(y)$. Hence, $\hat{y}=\hat{b}_{1}^{-1}\left(\hat{b}_{2}(x)\right)<y$.
} 
provide equilibrium bidding strategies first when the seller never reveals his signal and thereafter when he always reveals his signal. In each case, the strategies can be derived by employing appropriate versions of the equilibrium equations given in Section 3. ${ }^{9}$ Note that because $m_{2}(x, y, z)=x$, it is a dominant strategy for an agent to bid his signal on his second unit. ${ }^{10}$ Thus, whether or not the seller reveals his signal, we will have $\hat{b}_{2}(x)=x$ in equilibrium. Thus, in what follows it remains only to derive the equilibrium first-unit bid.

We remark here that once dominated strategies are eliminated, the equilibrium outcomes in the two informational settings considered below are unique. Consequently, although there are other undominated equilibrium strategies, they all yield the same revenues for the seller as the strategies we display below.

\subsection{When the Seller's Signal is Never Revealed}

Consider the following bidding strategy for an agent: For all $x \in[0,2]$

$$
\begin{aligned}
& \hat{b}_{1}(x)=2 \\
& \hat{b}_{2}(x)=x .
\end{aligned}
$$

We wish to demonstrate that these constitute an equilibrium. As remarked above, because $\hat{b}_{2}(x)=x$ is dominant, it remains only to show that the first-unit bid above is optimal. First, note that $E(Z \mid X=x, Y=y)=\frac{\min (x, y, 1)+\max (x, y, 1)}{2}$. Second, note that according to the equilibrium strategies, for every $x$ and $y$, each agent wins one unit of the good and pays the other agent's signal. Thus, it suffices to show that conditional on every $x$ and $y$, each agent's expected first-unit value exceeds the other agent's signal. That is, it suffices to show that for every $x$ and $y \in[0,2]$

$$
E\left(m_{1}(X, Y, Z) \mid X=x, Y=y\right) \geq y .
$$

\footnotetext{
${ }^{9}$ To keep the analysis of Sections 2 and 3 as simple as possible, we implicitly assumed there that the equilibrium first and second-unit bidding functions had the same image. As remarked in footnote 3 one can indeed impose enough structure on the marginal valuation functions so that this is the case. However, equilibria can be shown to exist without these additional restrictions as well, but the pair of bidding functions then need not have the same image. Nonetheless, the resulting equilibrium equations remain closely related to those given in Sections 2 and 3, and it is these, more general, equilibrium equations which yield the equilibrium bidding functions displayed below.

${ }^{10}$ Indeed any strategy in which either of the two bids is sometimes below the agent's signal is dominated by one in which both bids are never below the agent's signal.
} 
That this holds can be seen as follows:

$$
\begin{aligned}
E\left(m_{1}(X, Y, Z) \mid X=x, Y=y\right)=x+\frac{2}{3} y+\frac{2}{3} E(Z \mid X=x, Y=y) \\
\quad=\left(x+\frac{2}{3} y\right)+\left(\frac{\min (x, y, 1)+\max (x, y, 1)}{3}\right) \\
\geq \frac{2}{3} y+\frac{1}{3} y \\
=y .
\end{aligned}
$$

We conclude that the strategies given in (??) constitute a symmetric, non-decreasing equilibrium.

Note that because each agent always receives one unit, the two losing bids are the two second-unit bids. Consequently, when the seller's signal is never revealed, the seller's revenues are $x+y$ for each $x$ and $y$.

\subsection{When the Seller's Signal is Always Revealed}

Consider the following bidding strategy for an agent: For all $x, y, z \in[0,2]$

$$
\begin{aligned}
& \hat{b}_{1}(x ; z)=3 x+2 z \\
& \hat{b}_{2}(x ; z)=x .
\end{aligned}
$$

Once again, it suffices to show that the first-unit bid is optimal for every $x, y$, and $z$, given the opponent's bidding behavior. To see that this is indeed the case, note that given signals $x, y$, and $z$, agent 1 (with signal $x$ ) wins a first unit if and only if

$$
3 x+2 z=\hat{b}_{1}(x ; z)>\hat{b}_{2}(y ; z)=y .
$$

But the above inequality holds if and only if

$$
m_{1}(x, y, z)=x+\frac{2}{3}(y+z)>y=\hat{b}_{2}(y ; z) .
$$

Consequently, agent 1 wins a first unit precisely when his value for it exceeds what he must pay for it. We conclude that these strategies constitute a symmetric nondecreasing equilibrium. 
Now, because the seller's revenues are determined by the two lowest bids among the four submitted, his revenues when he always reveals his signal are equal to the sum of the lowest two among

$$
3 x+2 z ; x ; 3 y+2 z ; y,
$$

for every $x, y, z$.

\subsection{Hiding Information Yields Strictly More Revenue}

It is now easy to see that the linkage principle fails. Indeed, not only are the seller's revenues strictly larger on average when he hides rather than reveals his signal, hiding his signal dominates revealing it for every realization $x, y, z$.

To see this, fix $x, y$, and $z$. A seller who always hides his signal receives revenues equal to $x+y$, while a seller who always reveals his signal receives revenues equal to $\min (x+y, 4 x+2 z, 4 y+2 z) \leq x+y$. Moreover, the inequality is strict with positive probability. ${ }^{11}$

\section{Discussion}

As Milgrom and Weber (1982) have shown, there is a close connection between the linkage principle and the tenet that expected revenues in an ascending auction dominate those from a Vickrey auction. Indeed, the latter result is a corollary of the former in the single-unit setting (see Milgrom and Weber (1982), Theorem 11). This is because in an ascending auction, the last two remaining bidders observe the prices at which all others have dropped out. As pointed out by Milgrom and Weber, the implicit information so revealed about the others' signals plays the same role as would information revealed by the seller. Consequently, if revealing information is revenue enhancing for the seller (as the linkage principle implies in the single-unit setting), then the ascending auction dominates the Vickrey auction.

Now, although we have shown that the linkage principle does not extend to the multi-unit setting, this does not immediately imply that an ascending version of a multi-unit auction might fail to dominate the multi-unit Vickrey auction. However, it does raise serious concerns in this regard. One of the main difficulties

\footnotetext{
${ }^{11}$ In fact, the inequality is strict precisely when $\epsilon_{x}>4 \theta+3 \epsilon_{y}+2 \epsilon_{z}$ or $\epsilon_{y}>4 \theta+3 \epsilon_{x}+2 \epsilon_{z}$.
} 
in addressing this issue is the determination of equilibrium bidding behavior in a multi-unit ascending auction. ${ }^{12}$

We have focussed attention here on the Vickrey auction. On the other hand, Milgrom and Weber (1982) prove the linkage principle for both the single-unit Vickrey auction and the single-unit first-price sealed-bid auction. One might wonder whether the linkage principle remains true in the multi-unit setting for a first-price sealed-bid auction. We are very far from understanding the full complexities of the equilibrium bidding strategies in such an environment and so have rather little to offer in the way of insight here, except perhaps for the following observation. The failure of the linkage principle for the Vickrey auction, as we have shown, is due to the downward effect that the release of information has (on average) on winning bids. In a single-unit first-price sealed-bid auction, the release of information raises the winning bid on average. Needless to say, if information revelation raises all winning bids on average in the first-price multi-unit setting, the linkage principle would survive.

\section{Appendix}

Proof of Theorem ??. Suppose that $\hat{b}_{1}(\cdot)$ and $\hat{b}_{2}(\cdot)$ satisfy the hypotheses of the theorem. Since (??) and (??) are satisfied, it follows that $\hat{b}_{1}(\cdot)$ and $\hat{b}_{2}(\cdot)$ have the same image. Consequently, by (??) and (??), we have that for all $b$ in their common range:

$$
\begin{aligned}
& b=m_{1}\left(\hat{b}_{1}^{-1}(b), \hat{b}_{2}^{-1}(b)\right), \text { and } \\
& b=m_{2}\left(\hat{b}_{2}^{-1}(b), \hat{b}_{1}^{-1}(b)\right)
\end{aligned}
$$

Now, consider a state, $(\bar{x}, \bar{y})$, in which agent 1 wins precisely one unit. That is

$$
\begin{aligned}
& \hat{b}_{1}(\bar{y})>\hat{b}_{2}(\bar{x}), \text { and } \\
& \hat{b}_{1}(\bar{x})>\hat{b}_{2}(\bar{y})
\end{aligned}
$$

\footnotetext{
${ }^{12}$ Further concerns are raised by the examples of Frutos and Rosenthal (1997). In particular, they show that when bidders demand only one of two identical items, and the items are auctioned sequentially, the seller may raise more revenue by not revealing the winning bid on the first item.
} 
By replacing $b$ in (??) by $\hat{b}_{1}(\bar{y})$, we obtain

$$
\begin{aligned}
\hat{b}_{1}(\bar{y}) & =m_{2}\left(\hat{b}_{2}^{-1}\left(\hat{b}_{1}(\bar{y})\right), \bar{y}\right) \\
& \geq m_{2}(\bar{x}, \bar{y}),
\end{aligned}
$$

where the inequality follows from (??). Consequently, agent 1 does not wish to increase his second bid so as to win a second unit.

Similarly, by replacing $b$ in $(? ?)$ by $\hat{b}_{2}(\bar{y})$, we obtain

$$
\begin{aligned}
\hat{b}_{2}(\bar{y}) & =m_{1}\left(\hat{b}_{1}^{-1}\left(\hat{b}_{2}(\bar{y})\right), \bar{y}\right) \\
& \leq m_{1}(\bar{x}, \bar{y})
\end{aligned}
$$

where the inequality follows from (??). Consequently, agent 1 does at least as well by winning at least one unit as compared to winning neither unit. Thus, in all such states, agent 1 cannot improve his payoff.

Next, consider a state, $(\bar{x}, \bar{y})$, in which agent 1 wins both units. That is,

$$
\hat{b}_{2}(\bar{x})>\hat{b}_{1}(\bar{y})
$$

By replacing $b$ in (??) by $\hat{b}_{1}(\bar{y})$, we have

$$
\begin{aligned}
\hat{b}_{1}(\bar{y}) & =m_{2}\left(\hat{b}_{2}^{-1}\left(\hat{b}_{1}(\bar{y})\right), \bar{y}\right) \\
& \leq m_{2}(\bar{x}, \bar{y})
\end{aligned}
$$

where the inequality follows from (??). Consequently, agent 1's equilibrium bids are optimal in all such states.

Finally, consider a state, $(\bar{x}, \bar{y})$, in which agent 1 wins neither unit. That is,

$$
\hat{b}_{2}(\bar{y})>\hat{b}_{1}(\bar{x})
$$

Replacing $b$ in (??) by $\hat{b}_{2}(\bar{y})$ yields

$$
\begin{aligned}
\hat{b}_{2}(\bar{y}) & =m_{1}\left(\hat{b}_{1}^{-1}\left(\hat{b}_{2}(\bar{y})\right), \bar{y}\right) \\
& \geq m_{1}(\bar{x}, \bar{y})
\end{aligned}
$$

where the inequality follows from (??). Consequently, agent 1 cannot improve his payoff by attempting to obtain one or both units.

Thus, we have shown that in each state agent 1's bid is a best reply to agent 2 's bid (and vice versa, by symmetry). Consequently, a fortiori, the strategies constitute an equilibrium at the interim stage when the players know only their own signal. 


\section{REFERENCES}

Armstrong, M. (1996): "Multiproduct Nonlinear Pricing," Econometrica, 64, 5177.

Ausubel, L. (1997): "An Efficient Ascending-Bid Auction for Multiple Objects," mimeo, University of Maryland, College Park, MD.

Frutos, M. A. and R. W. Rosenthal (1997): "On Some Myths About Sequenced Common-Value Auctions," Boston University, ISP Discussion Paper \#77.

McMillan, J. (1994): "Selling Spectrum Rights," Journal of Economic Perspectives, 8, 145-162.

Milgrom, P. R., and R. J. Weber (1982): "A Theory of Auctions and Competitive Bidding," Econometrica, 50, 1089-1122.

Vickrey, W. (1961): "Counterspeculation, Auctions and Competitive Sealed Tenders," Journal of Finance, 16, 8-37. 